\title{
Planar Bragg Grating Sensors-Fabrication and Applications: A Review
}

\author{
I. J. G. Sparrow, ${ }^{1}$ P. G. R. Smith, ${ }^{1}$ G. D. Emmerson, ${ }^{1}$ S. P. Watts, ${ }^{1}$ and C. Riziotis ${ }^{2}$ \\ ${ }^{1}$ Stratophase Ltd, Unit A7, The Premier Centre, Premier Way, Romsey SO51 9DG, UK \\ ${ }^{2}$ Theoretical and Physical Chemistry Institute, The National Hellenic Research Foundation (NHRF), \\ 48 Vassileos Constantinou Avenue, 11635 Athens, Greece
}

Correspondence should be addressed to I. J. G. Sparrow, ian.sparrow@stratophase.com

Received 5 April 2009; Accepted 13 July 2009

Recommended by Valerio Pruneri

\begin{abstract}
We discuss the background and technology of planar Bragg grating sensors, reviewing their development and describing the latest developments. The physical operating principles are discussed, relating device operation to user requirements. Recent performance of such devices includes a planar Bragg grating sensor design which allows refractive index resolution of $1.9 \times 10^{-6} \mathrm{RIU}$ and temperature resolution of $0.03^{\circ} \mathrm{C}$. This sensor design is incorporated into industrialised applications allowing the sensor to be used for real time sensing in intrinsically safe, high-pressure pipelines, or for insertion probe applications such as fermentation. Initial data demonstrating the ability to identify solvents and monitor long term industrial processes is presented. A brief review of the technology used to fabricate the sensors is given along with examples of the flexibility afforded by the technique.
\end{abstract}

Copyright () 2009 I. J. G. Sparrow et al. This is an open access article distributed under the Creative Commons Attribution License, which permits unrestricted use, distribution, and reproduction in any medium, provided the original work is properly cited.

\section{Introduction}

A wide range of optical sensing technologies exist and are subject to intensive development due to a number of driving factors. In the fields of process control and automation for example, there is a desire to monitor concentrations and compositions in real time without the risk of damaging or contaminating high-value product. It is common for such applications to occur in volatile or flammable environments where spark-free or intrinsically safe technology is a prerequisite. Whilst there are many fields with disparate motivations and sensing requirements, they do in many ways share a common goal, that of rapid, accurate, and safe detection in a potentially harmful environment.

Existing review papers discuss the broad range of optical sensing technologies [1] and the motivations for using an integrated optical format in terms of compatibility with microfluidics [2]. In this paper we concentrate on a review of results on planar Bragg grating sensors which are a recent addition to the field and offer attractive advantages.

A detailed description of the range of different techniques and technologies available from the generic class of "optical sensors" would form an extensive review and could include a vast array of applications from particle counting to vibration detection. Therefore the various optical sensor technologies may be segmented in a wide number of ways, but it is helpful to distinguish between sensors in which the light does not physically pass into the measurand material (such as a Bragg grating sensor for strain) and sensors in which the optical field does pass into the measurand. In this paper we are concerned with the latter type of device. It is further useful to consider the means by which the light interacts; this may be either through the refractive index of the measurand or via an absorption or other energy exchanging interaction with the measurand. In that sense we can choose to characterise both types of linear properties of the light interacting in the material via a complex refractive index $\left(n^{*}=n-i \kappa\right)$ where $n$ is the real index at a particular wavelength and $\kappa$ represents the absorption. There are, of course, nonlinear interactions too, such as the Raman effect-which are outside of the scope of this paper.

By thinking in terms of the complex refractive index we can choose to classify techniques into either ones that make use of the imaginary part $(i \kappa)$ such as absorption 
spectroscopy, and those that make use of the real part of the index $(n)$ such as refractometry. In these terms we see that absorption spectroscopy can be viewed as investigating how $(i \kappa)$ varies with wavelength. However, in this paper we are primarily interested in those techniques that make use of the refractive index properties of the measurand, and particularly techniques in which the light interaction in the sensor and measurand modifies the modal properties of the light. A modal picture is familiar in fibre optics where a mode represents a solution to the laws of electromagnetic propagation that is constant in form along an invariant refractive index structure. This modal concept is distinct from refractive index properties (such as are used in a refractometer) in which the light propagation is effectively free space like with refraction occurring at a set of discrete boundaries. Thus from the huge possible range of sensor technologies we are led to consider firstly those that primarily sense the real part of the refractive index and then specialise to those techniques in which modal interactions are used.

Within this category of modal devices the most familiar devices make use of surface plasmon resonance [3] and provide a well-established technology $[4,5]$. Such plasmon devices are well established in literature; for a recent review of plasmonics the book by Maier [6] provides a wealth of information. Plasmon-based sensors are used in a number of commercial instruments produced by companies such as Biacore, Biosensing Instruments Inc, Sensata, and ICX Nomadics. In a Plasmon type sensor the light propagation and modal properties are strongly dependent on the properties of a thin film of a metallic conductor (most commonly gold), in which the modal coupling properties are modified by the refractive index of the surrounding dielectric (the measurand).

In contrast to SPR sensors, the types of devices in this paper make use of dielectric waveguides in which there are no metallic elements and in which the modal properties are dominated by the real part of the refractive index of the waveguide and of the measurand. The most familiar format for such a device is an optical fibre sensor. A recent review article can be found [7] which covers the whole area of fibre sensing. In this review we are specifically interested in devices in which guidance occurs by total internal reflection in a higher index core, and where the waveguide structure is processed to allow light to interact with a measurand fluid. This interaction causes a change in optical path length, which can be sensed in a number of ways but typically is either interferometric or via a change in the response of a grating structure. More recently, researchers have started to exploit the advantages of planar integration as a way to allow enhanced functionality devices to be made in which microfluidics and multiple sensor elements can be incorporated into a single device. Such devices have a common physical operating principle, in that they all operate by having a dielectric waveguide in which the propagating mode is allowed to partially interact with the measurand, and where the optical path change associated with that interaction is measured. For example work by Heideman et al. [8] describes the operation of a Mach-Zehnder sensor which measures fringe changes in the interferometer output; in contrast early work by Tiefenthaler [9] used a surface grating to measure water absorption on a planar waveguide. More recently work by Schroeder et al. [10] showed how multiple gratings at different operating wavelengths may be used to measure and correct for temperature variation and also gain information on the variation of refractive with wavelength; however, this device used a side-polished fibre embedded in a block, which is not simple to fabricate.

A relatively small number of Bragg grating-based devices have been considered and implemented in planar form. They have been demonstrated in a variety of different material platforms such as in polymers [11-14], Sol-gel systems [15], Silicon-on-Insulator SOI [16, 17], Lithium Niobate [18], and Silica-on-Silicon $[19,20]$.

The wave-guiding and grating structures in Bragg-based optical sensors have been fabricated with a number of approaches leading to sensing elements with ridge waveguides [21], UV written waveguides and gratings [19, 20], corrugated/etched Bragg gratings [22, 23], or even Bragg gratings through selective precipitation of nanoparticles [24].

However a very limited number of designs have been proved viable in terms of commercialisation. Recently, Stratophase Ltd has commercialised a direct UV writing technology following its original development at the University of Southampton $[19,20]$. The method allows the inscription of waveguides and gratings onto planar substrates. This technique enjoys the benefits of planar integration and ease of applying microfluidics and also makes use of telecommunication grade single mode fibre components and measurement technology allowing for tremendous refractive index sensitivity while exploiting the temperature compensation advantages first demonstrated by Schroeder et al. [10].

Moving on from consideration of the physical mechanisms, in the context of the work presented here, perhaps the most widely used tool is the benchtop refractometer upon which samples taken from processing steps are analysed in a lab to determine solution concentrations or sugar, alcohol, or solvents. Inline variants of this style of equipment have started to reach the market in recent years so that offline measurements may be replaced with at-line or inline measurements to save time, money and reduce safety and contamination concerns. Generally these tools require an electrical signal at the point of measurement which can be problematic in volatile environments which require intrinsically safe equipment to minimise the risk of explosion.

This paper presents a review of recent advances that have been made in the development of an optical sensor that has proven to be suitable in the application areas described above. The sensor, an evanescent wave device based on planar Bragg gratings, offers both the required sensitivity for concentration measurements and process monitoring and is also suited to industrial environments where robust and reliable devices must be installed. The all optical measurement means that there is no ignition risk, making the technology highly suitable for volatile environments. Additionally, because the underlying principles are the same as those used in the field of telecommunications, multiple 
devices may be networked and multiplexed over very large distances. Multiple devices located at separate and distant locations can easily be monitored from a single analysis base station to maximise convenience and minimise cost.

To describe the sensing technology, an overview of the fabrication technique and its advantageous features is given. This is followed by an analysis of the device sensitivity to refractive index and temperature. To complete the presentation two examples of industrial applications are given.

\section{Background}

The core technology of the sensors discussed here is that of the Bragg grating, a structure that has been known for decades and has always been recognised as having the potential for use as a sensing element. Most commonly employed in optical fibres, the Bragg grating reflects optical wavelengths according to the following relation:

$$
\lambda_{\mathrm{B}}=2 \Lambda n_{\mathrm{eff}}
$$

where $\lambda_{\mathrm{B}}$ is the Bragg wavelength at which maximum reflectivity occurs. $\Lambda$ provides the period of the refractive index modulation that defines the grating. The effective index of the waveguide that contains the Bragg grating, $n_{\mathrm{eff}}$, is a combined refractive index of the core and cladding that the optical mode interacts with.

From this equation it can be seen that as the material surrounding the Bragg grating changes, variation in the effective refractive index causes the reflected wavelength to shift. This forms the basis of the use of Bragg gratings as sensors and is shown conceptually in Figure 1.

The specific devices that will be outlined in the subsequent discussion use the technique of UV writing. This approach is highly flexible and has been highly refined for the creation and postfabrication trimming [25] of optical devices suitable for, amongst other applications, the telecommunications industry.

The sensors described here are fabricated using a unique extension to the UV writing technology known as Direct Grating Writing which simultaneously creates a waveguide and a Bragg grating in a planar substrate.

Early work on the conversion of the UV written substrates into liquid sensors has been presented along with demonstrations of their use as tunable filters and refractometers. Whilst this early work highlights some of the opportunities for such devices, the level of development was not initially suited towards full commercial exploitation.

Such planar Bragg grating devices are appealing for sensing applications for several reasons.

(1) Multiple wavelengths may be used offering the possibility for analyte identification through optical dispersion measurements and also providing a range of evanescent field penetration depths which may provide additional information on the dimensions of biological entities.

(2) Multiple separate sensing regions may be incorporated onto a single sensor chip. This can be an

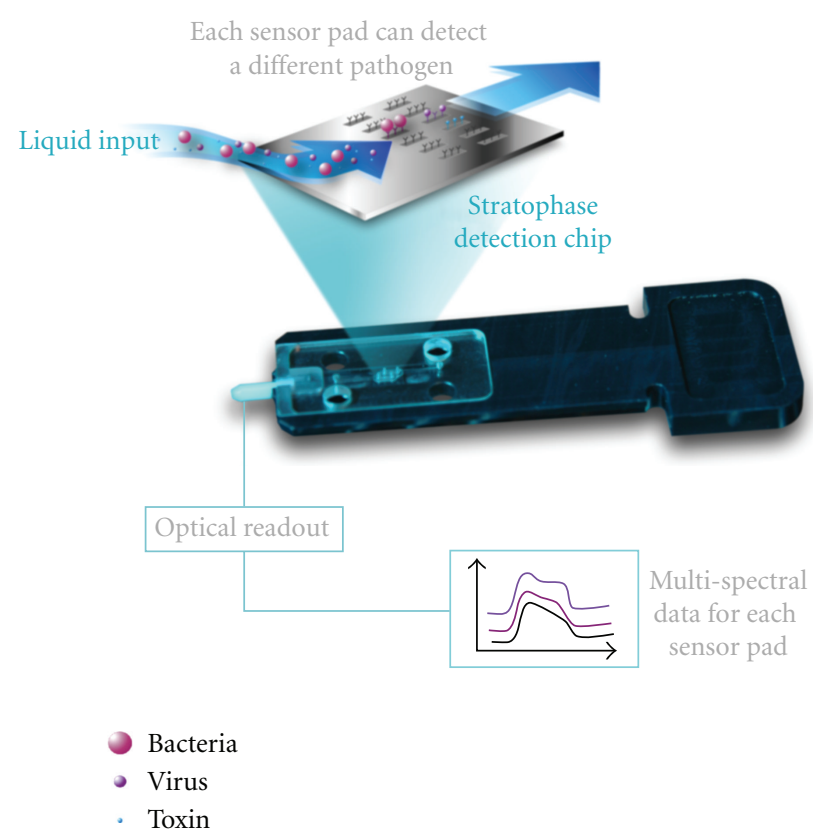

Figure 1: Representation of the interaction between liquid and a Stratophase sensor chip that is mounted in a microfluidic cell. In this case a functionalised chip for the purposes of specific biological detection is shown.

advantage particularly in immunoassay-based biodetection where it is advantageous to test for multiple different targets simultaneously without the need for duplication of equipment or time delays.

(3) The monolithic silicon chip-based design is robust, requires no electrical signal, is resistant to a wide range of chemicals, and is thus suitable for deployment in a wide range of environments.

In order to get the maximum possible performance from a Bragg sensor the optical interrogation method is critical. Many industrial applications require multiple measurements to be made 24 hours per day, and so relatively high capital cost can be tolerated as the cost is shared. For top end performance the interrogation can come at a relatively high financial cost although technology improvements and commercial competition provide a strong drive for cost reduction over time. Additionally, the devices presented here are designed to operate in the telecommunications wavelength band. As such it is possible to have sensors positioned at up to several kilometre distances from the optical source. More advantageous still, signals from multiple sensors at different locations may be multiplexed in such a way as to have many sensors all monitored by a single read-out unit. Thus the cost per sensor, which is often more important than total cost, is dramatically reduced. Furthermore, the cost per measurement is lower still because of the opportunity to incorporate multiple sensing regions on a single chip.

In addition there is the possibility to integrate the sensing element with the interrogation system by deploying, for example, Bragg grating or Arrayed Waveguide Gratings 
[26-28] within the same chip, a technology that is highly compatible and readily available in the Silica-on-Silicon platform. This combination could lead to compact and selfcontained sensing systems.

\section{Fabrication of Sensors}

3.1. UV Writing. In a method similar to prior work [29] and shown in Figure 2, Bragg gratings and waveguides are written into a three-layer silica on silicon substrate using the DGW technique.

The UV writing process utilises the photosensitivity of Germanium doped silica. Exposure of the silica to UV light at $244 \mathrm{~nm}$ causes an increase in the refractive index. The three layer samples are fabricated by flame hydrolysis deposition such that the silica is deposited onto a silicon substrate. The middle layer is doped with germanium to provide a photosensitive layer that may be exposed to UV to raise the refractive index.

High-pressure hydrogen loading is used as a method of enhancing the photosensitive response immediately prior to UV exposure. Similar techniques are used in the fabrication of fibre Bragg gratings prior to UV exposure for grating inscription. The planar samples are placed into hydrogen at 120 bar for three days in order to allow hydrogen to diffuse through to the glass layer, that is, to act as the waveguide core. After hydrogen loading chips may be kept for many days in dry ice before UV writing. Once removed from cold storage they may be kept at room temperature for in excess of an hour before out-diffusion of hydrogen becomes a significant issue. The initial out-diffusion of hydrogen can result in a slight change of Bragg wavelength due to a reduction in photosensitivity over time. This may be compensated for with a well-controlled UV writing process if required. However in this application it is not the UV writing process that determines sensitivity to refractive index. It is in fact the later processing stages of etching and over-layer deposition described in Section 3 that determine overall performance. Thus long UV writing times may be used, and complex structures may be written without the need for any other special precautions.

Controlled, localised irradiation of the silica with a sufficiently high fluence after hydrogen loading allows welldefined waveguide structures to be created. For the work reported here, a frequency doubled argon-ion laser is used to produce the UV beam for irradiation. The beam is conditioned and focussed down to a five micron diameter spot in a fixed location. Samples for UV writing are mounted on an air-bearing translation stage which allows three dimensional movement of the photosensitive substrate to a spatial resolution better than $10 \mathrm{~nm}$.

The most direct method of creating a waveguide is to simply translate the sample at a constant speed under the UV beam at a constant power. However, to allow the inscription of both Bragg gratings and waveguides this simple arrangement is extended. Using a beam splitter and carefully positioned mirrors an interferometer is created such that the UV beam is recombined to create an interference pattern. This pattern, when incident on a photosensitive sample, will

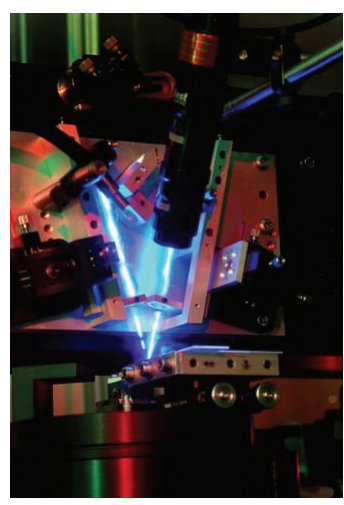

(a)

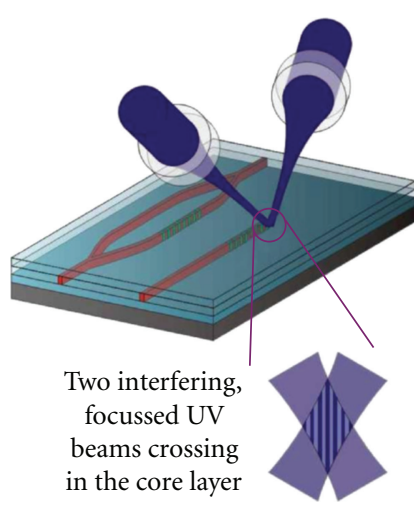

(b)
Figure 2: Photographic and diagrammatic representation of the UV writing process with crossing UV beams focussed onto the photosensitive substrate.

create a corresponding refractive index modulation in the core layer. The period of the interference pattern is set to be $530 \mathrm{~nm}$, a period which when combined with the refractive index of the core layer can be used to create a Bragg grating reflecting at $1550 \mathrm{~nm}$.

The interference pattern cannot simply be translated across the sample to create a Bragg grating. A static exposure would, in effect, create a very short Bragg grating of just a few grating planes in length. However, upon translating the sample this refractive index modulation would be "smeared out" in the direction of translation. This destroys the periodic refractive index modulation resulting in a uniform increase in the refractive index. Thus, translation of the sample beneath a continuous UV spot results in the formation of an unmodulated waveguide. To create, a Bragg grating the UV beam must be modulated as the sample is translated under the UV beams. Monitoring the position of the sample as it is translated and briefly turning on the UV beam every time the sample has moved by one grating period causes the pattern of refractive index modulation to be repeatedly written into the sample. This occurs in such a way that each pattern is shifted by precisely one period relative to the previous one. In other words, as the sample is translated a Bragg grating is written into the sample. To change from Bragg grating to waveguide the UV beam is simply returned to its former nonmodulating state. In this way, a single process may be used to write both waveguides and Bragg gratings into a sample in a single but highly flexible process. Accurate control of the UV switching is achieved by using the high precision position information from the translation stages to drive an acousto-optic modulator.

3.2. Grating Parameters. A number of parameters may be controlled in order to tailor the Bragg gratings and waveguides. The primary factor in UV writing is the fluence used to write the waveguide and grating structures. A higher UV power (or a lower translation speed) gives a higher fluence, resulting in larger changes in refractive index. 
The relationship between translation speed and the rate at which the UV beam is modulated plays an important role in the creation of Bragg structures. Variation of the duty cycle can be used to control the strength of the Bragg grating. Duty cycle refers to the percentage of time that the UV beam is turned on in the process of writing a Bragg grating. Previously, the modulation of the UV beam whilst the sample is translated was discussed. The duration of this modulation as a percentage of the Bragg period gives the duty cycle. A duty cycle of 100 percent results in a waveguide with no refractive index modulation whilst a duty cycle of 0 percent results in no refractive index change being written into the sample whatsoever.

It is desirable for the average refractive index of a Bragg grating to be close to, if not identical to, the waveguide at either end of the grating. Although the link between UV power and induced refractive index change is not perfectly linear, to a close approximation fluence and duty cycle may be used to index match waveguides and gratings very simply. A grating written with a 50-percent duty cycle with a given UV power must be translated under the UV beam at half the speed used for a waveguide written with the same power. This gives the same fluence and therefore approximately the same average refractive index in both the grating and waveguide. Similarly a 90 -percent duty cycle grating would be translated at 90 percent of the waveguide translation speed to "fluence match" the two structures. This simple relation is possible because the waveguides and gratings are being written into a "blank" substrate where no waveguide exists beforehand. In the case of fibre Bragg gratings this is slightly different as the gratings are added to a pre-existing waveguide, and so the average index of the waveguide and fibre cannot be the same unless special extra steps are taken.

Duty cycle may also be used to change the strength of the Bragg gratings written. Generally speaking, a higher duty cycle results in a lower contrast between the grating planes resulting in a more response with a more narrow bandwidth than one written with lower duty cycles.

An additional degree of flexibility may be achieved by modulating the UV beam at a rate very slightly different to the intrinsic period of the UV intensity modulation. The cumulative effect of the multiple UV exposures used to create a grating produces a grating with a period that is equal to the period between exposures, not the intrinsic period of the interference pattern. [30]. In this way, gratings may be written spanning hundreds of nanometer using exactly the same process.

Refinement of the UV writing process has allowed an immensely flexible technique for waveguide and grating production to be developed. Using specially developed software packages it becomes a simple matter to create scripts which when loaded into the UV writing system can produce straight and curved waveguides with sophisticated grating structures at multiple wavelengths in a single process. Not only can bespoke waveguide and grating designs be rapidly created without the need for expensive phase masks but also no clean room facilities are needed, making the overall infrastructure requirements low.

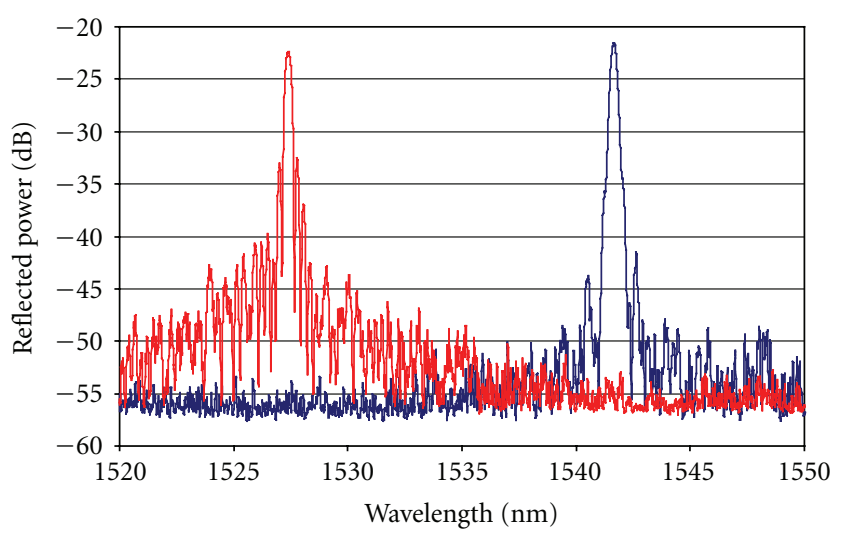

— Apodised grating
_ Uniform grating

Figure 3: Comparison of reflection spectra of uniform and apodised gratings.

Some examples of grating spectra that may be written using this process are given below. All structures were created using the same proprietary software package to create the required grating responses.

Figure 3 compares a straightforward uniform grating with another grating of similar properties but which has been apodised using a cosine squared function. The reduction in sidelobes is clear albeit at the expense of a slightly broader reflection peak.

As the gratings are written in a manner that is close to being plane by plane, it is straightforward to achieve high levels of control of the grating structure along its length. For example, phase shifts may be inserted in order to achieve a sharp dip in the reflection response which may in some cases be advantageous when determining the centre wavelength of peaks. Such a spectrum is shown in Figure 4.

Periodic spacing of phase shifts can be used to generate more elaborate grating structures which provide multiwavelength responses. An example of this is given in Figure 5, which is a demonstration of a superstructured grating providing over 15 wavelengths that may be used for sensing purposes over a $130 \mathrm{~nm}$ wavelength span. This grating, just $4 \mathrm{~mm}$ in length, opens up opportunities to measure refractive index over sufficiently wide ranges to allow dispersion characterisation and thus material fingerprinting to be performed.

This approach may be extended, as shown in Figure 6, to use multiple superstructured gratings that are interlaced such that they allow a greater spectral density of measurements to be performed from a compact device which is again written in a single step onto a single device that is just millimetres in dimensions.

Drawing on the technology of fibre Bragg gratings it should be possible, if required, to fully control the spectral properties of Bragg gratings through the use of novel design strategies employed in fibres [31] and then implement them through the DGW fabrication technique. This advantage gives this particular planar platform further flexibility to 


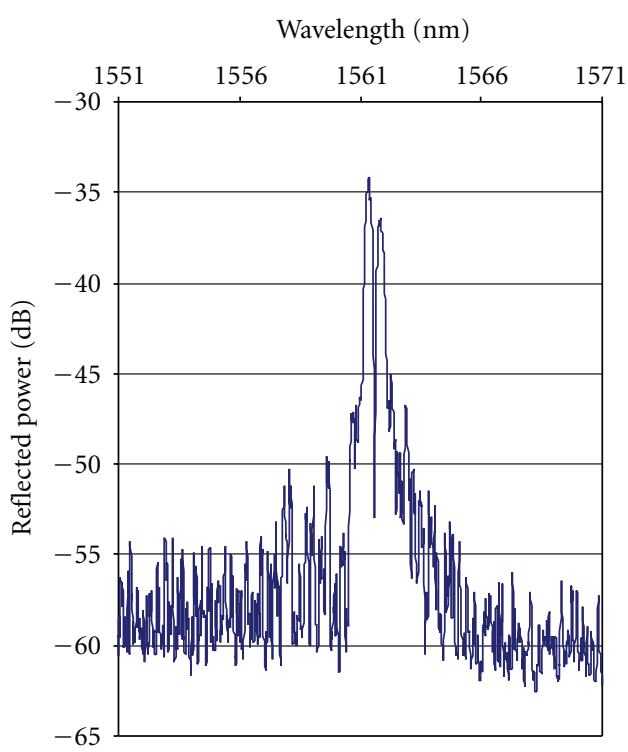

(a)

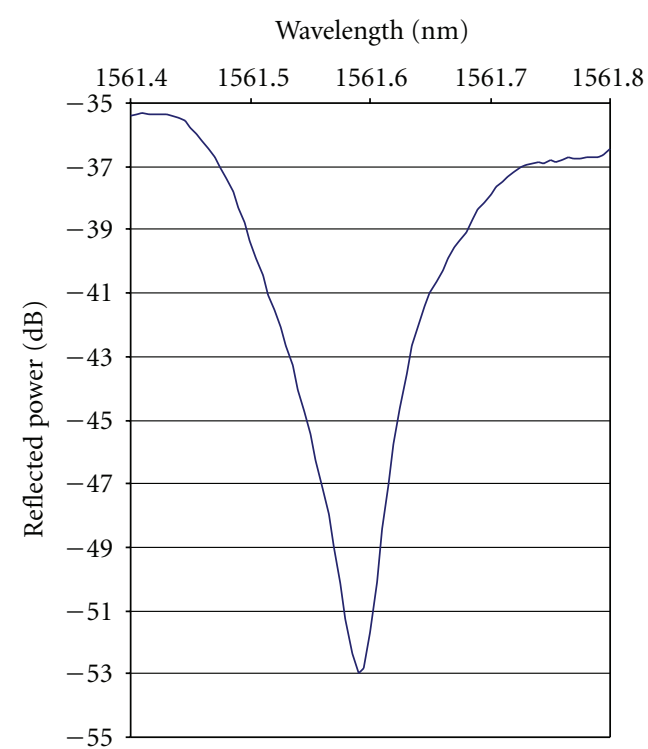

(b)

FIGURE 4: Example of a phase-shifted Bragg grating with a deep transmission dip written by DGW. The grating used here has a total length of $3 \mathrm{~mm}$.

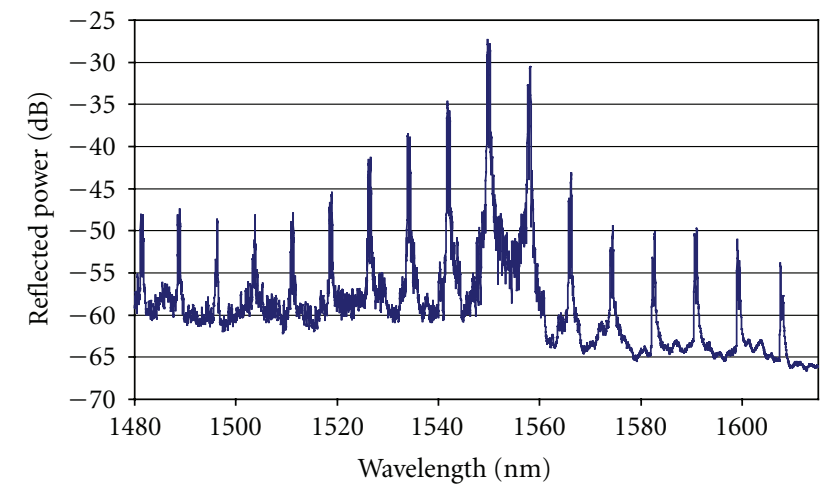

FIGURE 5: Wide span reflection spectrum of a superstructured grating written into a planar sensor chip.

develop customised purpose-oriented sensors combined in a compact integrated form.

3.3. Creating a Sensor. In their as-written state, the UV written samples are intrinsically sensitive to temperature or stress and can be used as sensors in a manner comparable to that widely used in fibre sensing [7]. To exploit the advantages of the planar geometry this temperature sensing ability may be combined with multiple liquid refractive index sensing regions. Conversion to a sensor uses relatively simple principles. The Bragg wavelength of a grating is determined by the effective index of the waveguiding structure in which the grating is defined. In other words the combined refractive indices of the waveguide core and cladding play a key role in setting the wavelength or wavelengths at which the Bragg grating operates. If we remove the upper cladding and replace

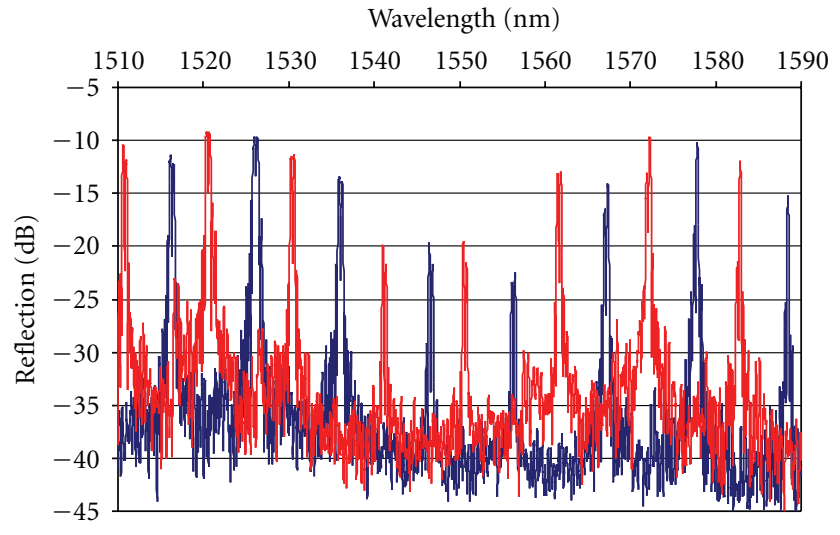

FIGURE 6: Reflection spectra of two interlaced superstructure Bragg gratings on separate waveguides. These allow multiple wavelengths to be measured in a single sensing region on a single chip.

it with something else, the effective index and thus the Bragg wavelength is changed. In this way, the device can now be made into a sensor. Liquid that is used to replace the upper cladding in the vicinity of the Bragg grating will control the Bragg wavelength. As the properties of that liquid change, so the Bragg wavelength changes.

The cladding over the sensor gratings is removed using a wet etch. The etchant is delivered to the silica surface using a microfluidic flow cell which allows the chemical to come into contact with only the areas of the chip where etching is required. The Bragg response is monitored throughout the etching process to ensure that the etch is allowed to proceed until sufficiently deep to ensure that the cladding is removed but that it is stopped before the response is degraded by 


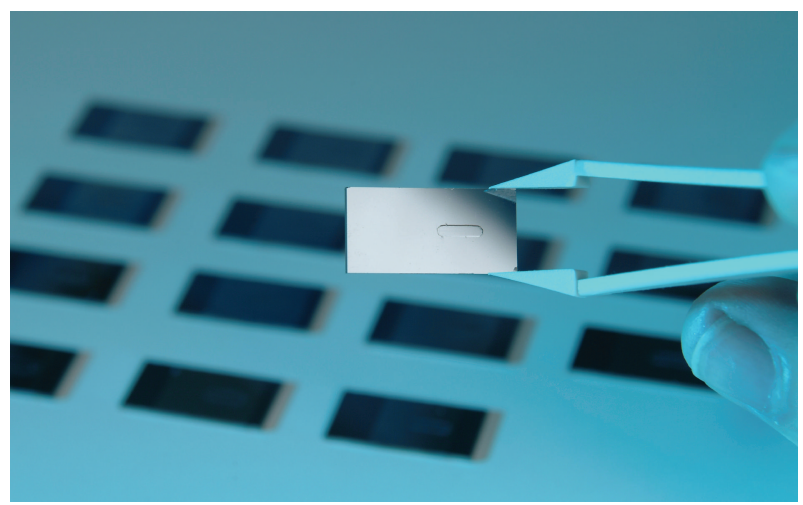

FIGURE 7: An etched sensor chip before fibre pigtailing. The sensing region is seen as a small oval towards the right hand end of the chip.

removal of the waveguide core. Temperature measurement gratings are left unetched such that they are immune to changes in the refractive index of any liquids above them.

Following etching, the penetration depth of the optical mode into the liquid analyte is relatively low. To extend the penetration further, a high-index overlayer may be applied to the etched surface [32]. This has the effect of lifting the optical mode up towards the analyte, resulting in a much higher penetration depth and a much greater sensitivity to refractive index. A number of methods and materials may be used to achieve this effect depending on the desired upper surface material and the required refractive index sensitivity. To date several materials have been utilised including, silica, silicon nitride, silicon oxynitride, fluorinated polymers, titania, zirconia, and alumina. The different materials all require different processing steps and results in different end products.

To perform measurements the etched and overlayered chips are pigtailed using single mode fibre, and optical spectra are obtained with the use of commercially available Bragg grating interrogators.

Figure 7 shows a photograph of a typical sensor chip after fabrication but before optical fibre pigtailing. The etched window containing the sensing region can be seen as a small oval to the right of the chip. Temperature measurement gratings are embedded in the left-hand side of the chip.

It should be noted that the process of etching the chip and subsequently adding a high-index overlayer brings about a very high level of birefringence in the Bragg grating section of the device. In many applications this would not be tolerable as it would cause large amounts of polarisation dependence. Here, the effect is sufficiently large that TE and TM modes can be independently resolved. This means that instead of requiring polarisation control to obtain a reliable mode of operation, the more simple route of using an unpolarised optical source can, if desired, be taken.

Similarly, whilst overall optical loss would in many applications be required to be minimal, it is of less concern here. It is critical to measure changes in Bragg wavelength as accurately as possible but this is not dependant on the optical power that is reflected.

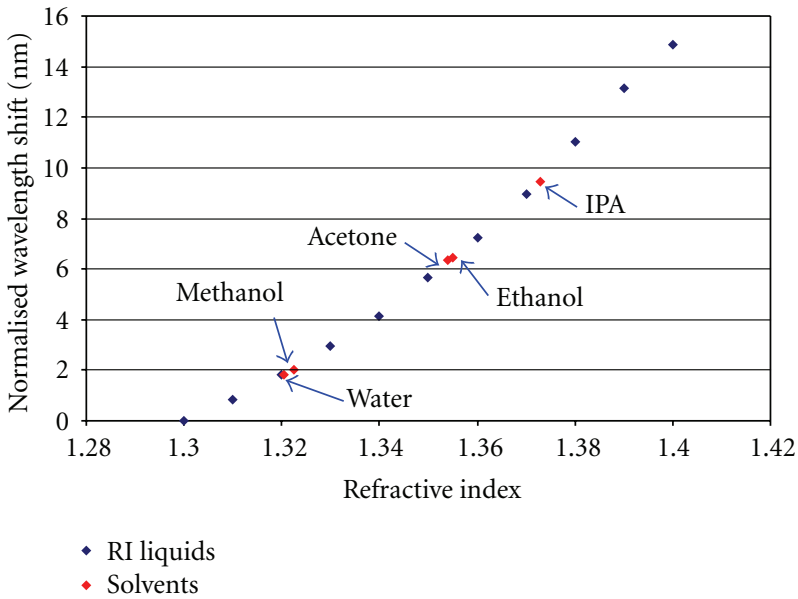

FIGURE 8: Sensitivity curve showing variation in Bragg wavelength with refractive index.

\section{Refractive Index Sensitivity}

The ability to detect changes in refractive index depends on two key aspects when using a Bragg grating device. Firstly and most obviously, the rate at which the Bragg wavelength varies with refractive index, the intrinsic sensor sensitivity, must be considered. Secondly, the resolution and accuracy to which the Bragg wavelength can be determined must be taken into account.

To address the first of these considerations, a simple sensitivity curve may be generated. Such a curve is shown in Figure 8 where Cargille refractive index liquids are used on the sensor chips, and the resultant Bragg wavelengths are measured. It must be noted that there is a very slight uncertainty in this data as the RI liquids are quoted at $589 \mathrm{~nm}$, not at $1550 \mathrm{~nm}$ used here. As a result of material dispersion, there will be small but currently unknown offset between the RI values quoted in this graph and their true values.

It is easy to see that over a refractive index span of 1.30 to 1.40 the Bragg wavelength shifts by approximately $15 \mathrm{~nm}$, giving rise to a particularly high sensitivity to refractive index. The sensitivity of this device increases at higher refractive indices as expected [20]. The gradient of the curve provides the sensitivity measured in refractive index units (RIUs) per nanometre. At an index of 1.31 the sensitivity shown is $92 \mathrm{RIU} / \mathrm{nm}$ rising to $155 \mathrm{RIU} / \mathrm{nm}$ at an index of 1.36. The upper end of the curve shown here gives a sensitivity of $193 \mathrm{RIU} / \mathrm{nm}$ at a refractive index of 1.39 . At analyte refractive indices much above 1.40 this particular device ceases to act as a waveguide. The combined effect of the high-index overlayer and the refractive index of the analyte means significant modal mismatch occurs as the light travels into the etched window region, and all of the optical mode is lost to the overlayer and analyte with the result that the Bragg signal is completely lost.

In addition to the RI liquid data shown on Figure 8, data points associated with several solvents are included. These data points were obtained by simply using the relevant 


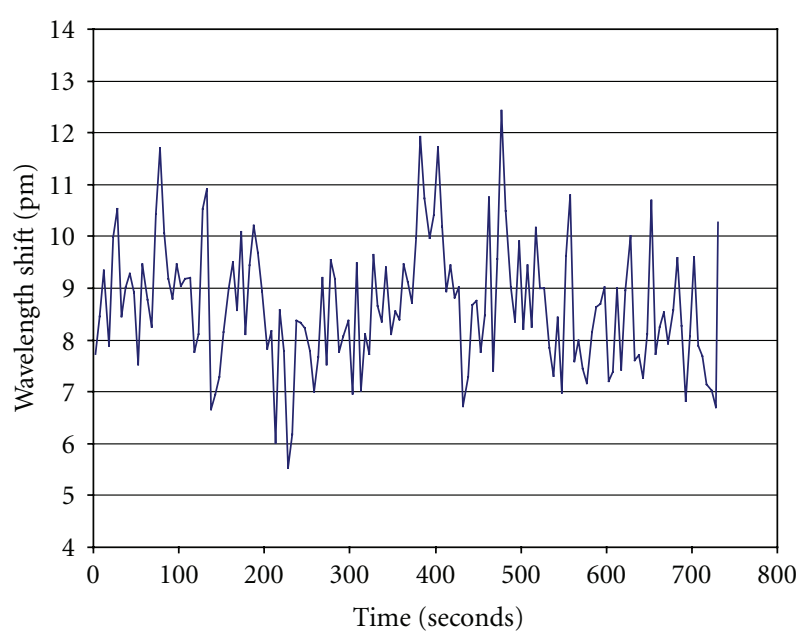

FIGURE 9: Steady-state Bragg wavelength determination showing subpicometre stability.

solvent as the analyte on the sensor. The measured Bragg wavelength allows the solvent data points to be interpolated onto the existing RI curve such that the measured RI of the solvents can be determined. Clearly the five solvents, namely, water, methanol, acetone, ethanol, and isopropyl alcohol, can all be easily resolved and thus identified by measurement of the Bragg wavelength. These liquids represent just a small subset of the liquids that may be monitored in this way.

The second factor in determining refractive index resolution is that of the measurement technique. Firstly, an optical spectrum must be obtained in an easy to manipulate format. Within this spectrum the Bragg reflection must be identified and the centre wavelength determined. A number of Bragg grating interrogators are available in an off the shelf configuration, typically operating in wavelengths between 1500 and $1600 \mathrm{~nm}$. The stability and repeatability of the interrogators is of obvious importance as is the reliability of the centre wavelength determination from the reflection spectrum.

Using a simple weighted average calculation on a reflection peak a quick and easy measurement of centre wavelength may be made. Figure 9 shows the result of such a calculation on a single Bragg reflection from a sample made according to the techniques outlined earlier. The chip was held at a constant $35^{\circ} \mathrm{C}$ using a PID temperature controller in a stable environment such that the Bragg wavelengths of the peaks would remain constant as no external changes were permitted. Measuring the Bragg wavelength for a period of time allows an estimate of the stability and the calculation to be made.

This data shows that over the 12 minute section in the graph the wavelength varies by a maximum of $0.7 \mathrm{pm}$ representing excellent stability. Each data point shown is the mean wavelength of 5 seconds worth of data collected from single Bragg reflection peak. A common method of quoting the stability is to multiply the standard deviation of the data set by three, a calculation which provides a stability of just $0.36 \mathrm{pm}$.
With the assumption that any change in wavelength greater than $0.36 \mathrm{pm}$ should be the smallest change in wavelength that can be resolved with this combination of chip, interrogator, and analysis technique, we can determine the refractive index resolution. Figure 8 allowed the sensitivity to refractive index to be measured at $193 \mathrm{RIU} / \mathrm{nm}$; so with a wavelength resolution of $0.36 \mathrm{pm}$ we obtain a refractive index resolution of just $1.9 \times 10^{-6}$ RIU. Further time-averaging may allow this to be further improved but this would come at the expense of data update rate which in many applications is an important parameter.

\section{Temperature Measurement}

As mentioned earlier, the UV written chips may be used as temperature sensing devices as well as refractive index sensors by choosing to leave one or more of the Bragg gratings unetched. In this case thermal expansion of the silica brings about a change in the Bragg period and therefore in the Bragg wavelength.

Using a temperature controlled and stabilised environment a sensor chip was ramped up and down between multiple temperatures and allowed to stabilise at five degree intervals. Both the Bragg wavelength and the actual chip temperature were recorded throughout the process. Temperature was measured using a $100 \Omega$ PRT monitored using a Measurement Computing USB-Temp module. This data is shown in Figure 10 with the data points between stable temperatures being removed for clarity. The steps in output as the temperature was ramped up and down are easily seen. In order to see the relationship between temperature and wavelength the data is replotted in Figure 11 where a linear relationship between temperature and wavelength is clear. Whilst this graph appears to show only nine data points, there is in fact over twenty five thousand points plotted. The points are very closely clustered due to the stability of the temperature and therefore wavelength.

The data in Figure 11 yields a temperature coefficient for this unetched grating of $0.011 \mathrm{~nm}$ per ${ }^{\circ} \mathrm{C}$, which when using the wavelength resolution of $0.36 \mathrm{pm}$ derived earlier gives a temperature resolution of just $0.03^{\circ} \mathrm{C}$. This resolution is easily equal to that obtainable by many electrical methods and is made more attractive as the optical nature of the measurement will not be compromised in electrically noisy environments which may degrade the signals from PRT and thermistor type devices. Additionally, this temperature measurement technique is entirely compatible with the refractive index sensing technology as well as being safe for use in volatile or flammable environments.

\section{Real World Measurement Techniques}

One of the great challenges in creating a sensor technology is the conversion from development laboratory experiment into a robust, easy to use package suitable for real world applications. The packaging needs to meet end-user requirements and be sufficiently robust to allow installation by 


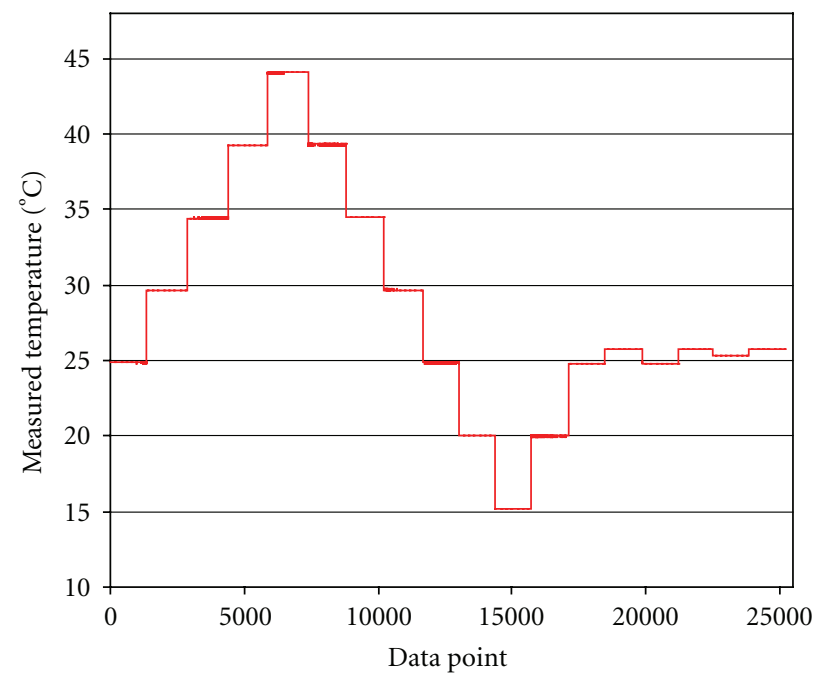

(a)

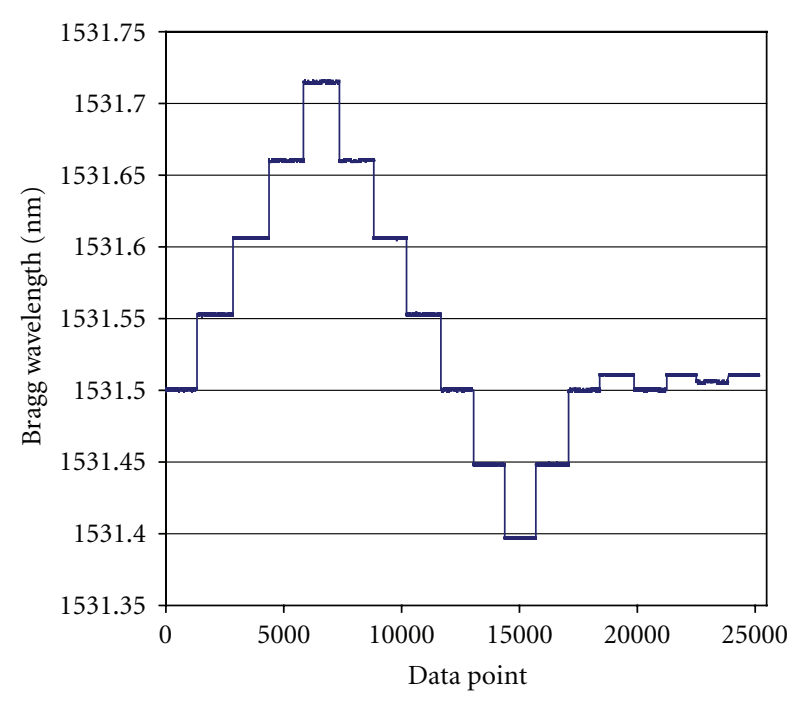

(b)

FIGURE 10: Variation in temperature (left-hand graph measured using PRT) and corresponding Bragg wavelength (right-hand graph) over time. Data points recorded at a rate of $2 \mathrm{~Hz}$.

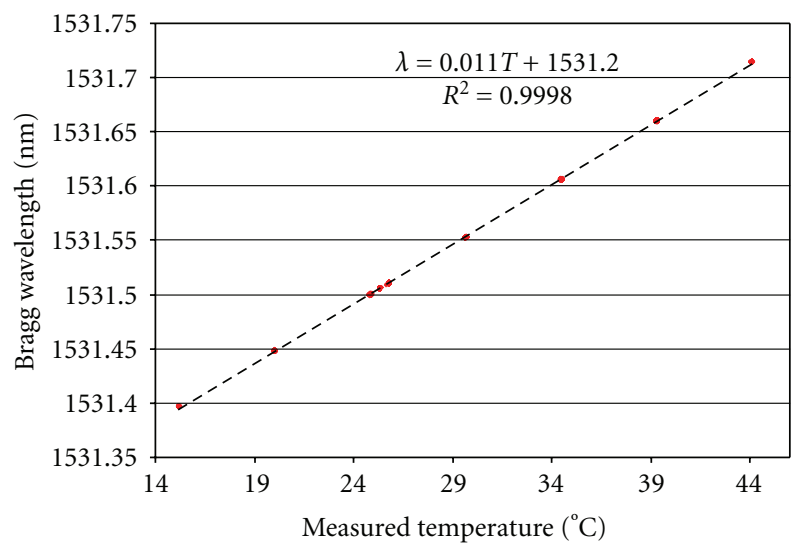

FIgURE 11: Relationship between temperature and Bragg wavelength. Note that over twenty five thousand data points are plotted on this graph but that high precision temperature measurement results in data points being closely clustered.

unskilled workers but retain the performance of the laboratory prototypes. To this end, the sensor technology discussed above has been integrated into a variety of application specific packages which allow the liquids for analysis to be easily delivered to the sensing region.

6.1. High-Flow Industrial Process Control Flow Cell. Monitoring pipelines in a process can be an invaluable tool for protecting the overall process integrity in many applications. An unknown or uncontrolled concentration of reagents entering a process at any point may severely compromise or totally write-off a complete processing batch. In order to allow pipeline contents to be measured in situ and in real time, Stratophase has defined a series of sensor chip products (Figure 12) that have been designed into a robust stainless

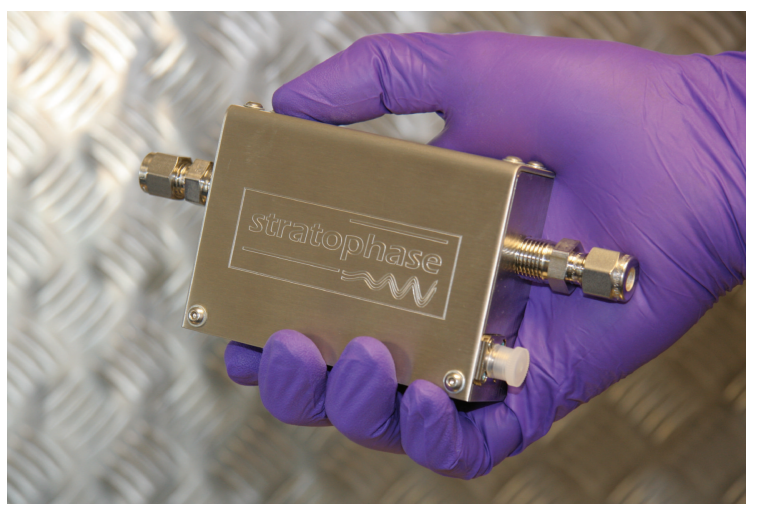

FIGURE 12: Industrial sensor cell for high-volume flow applications.

steel unit designed for incorporation into high-flow, high pressure pipelines. The industrial process control (IPC) flow cells are designed to accept standard 1/4" Swagelok fittings and pipes and allow liquid pressures up to 6 bars to be used.

6.2. Reaction Vessel Insertion Probe. Many applications, particularly those requiring fermentation processes for pharmaceutical, biofuel, or food and beverage must monitor their reactions in large reaction vessels or in multiple bench-top bioreactors. In these applications, a flow cell configuration is not always suitable as it requires a portion of the liquid to be tapped off from the main tank and pumped through a separate sensing loop. Instead, insertion probes are commonly used to allow sensors to be pushed through the walls or lids of the reaction vessels. A common solution uses a standard form-factor, typically with a Pg13.5 thread to allow sensors to be screwed into standard ports fitted to the fermenters or reactors. A probe-housing compatible with standard ports has been designed to allow the planar 


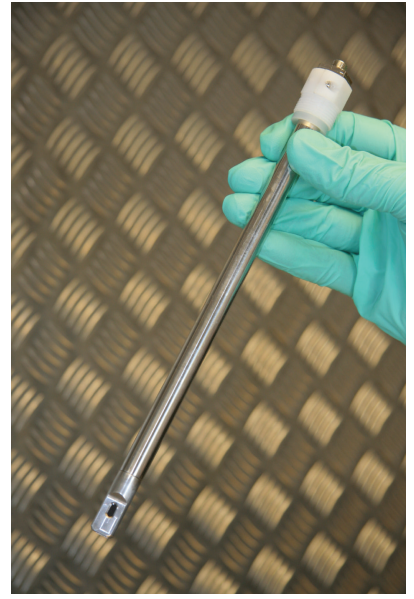

FIgURE 13: Probe sensor for in-vessel monitoring applications.

Bragg sensors discussed here to be used in an insertion probe format (Figure 13). The probes may be used in static or retractable housings and require only an optical fibre connection to access the sensor data.

\section{Example Applications}

Using the inline flow cell and insertion probe formats, the planar Bragg grating devices are capable of monitoring many aspects of process evolution over many weeks. Whilst the focus of this paper has so far been on the sensitivity to refractive index, it is more common to require an insight into changes in a process due to concentration variation or biological growth rather than in absolute refractive index. Even the smallest change in the properties of a liquid will result in a refractive index change regardless of whether the change is due to temperature, concentration, or other reaction events. By measuring both temperature and RI simultaneously and detailed picture of process evolution may be obtained.

7.1. Real Time Monitoring of Fermentation Processes. The process of fermentation using yeast to convert sugar to alcohol is as old as it is commercially important. The presence of sugars, nutrients, and final alcohol content are the key factors of interest, and the variation of these may be observed through refractive index change. To demonstrate this capability the IPC flow cell described above was plumbed into a standard fermentation process using a pump to circulate the fermenting liquid over a sensor. Tracking the variation in Bragg wavelength over a number of weeks reveals clear events that correlate to distinct phases of the process. Figure 14 shows how the sensor output varies with the process evolution over several weeks. The refractive index output of the sensor is shown on the left-hand axis and is plotted in units of nanometres and provides the change in Bragg wavelength over time. These units are used rather than conversion to refractive index units as it is overall insight into the fermentation process that is desirable, not the absolute refractive index at any given moment. In contrast, the output of the sensor that provides temperature has been calibrated into degrees $\mathrm{C}$.

During the initial stages it is clear that the Bragg wavelength drops steadily due to the reduction in sugar concentration as it is consumed by the yeast. Following this initial activity the rate of change of concentration decreases as more and more of the sugar is consumed, and the sensor output indicates that a steady state has been achieved. Between about 500 and 700 hours of process time several fining agents are added to the brewing liquid in order to assist in separating out the yeast and from the liquid product of interest. This causes step changes in both positive and negative directions as the decrease in suspended particles is reduced and as further chemicals are added, respectively. In the final stages, additives to improve flavour and mouthfeel are added to the liquid, causing a distinct change in the sensor output.

The lower trace on the graph, which corresponds to the right-hand axis, shows the optically measured temperature variation and allows events in the liquid sensor trace to be decoupled from temperature effects. For example, at about 500 hours of processing time a fault in the environmental temperature controls caused an oscillation of several degrees in the temperature. This is reflected in the upper fermentation trace. Other key events in the fermentation trace are known to be "real" effects of the process and not due to environmental changes as they are not mirrored by the temperature reference data.

These steps show that the processes may be monitored continuously in real time. Perhaps more importantly, they also show that additive or sugar concentration may be measured throughout the process such that remedial action may be immediately taken should the process deviate from the required path.

7.2. Industrial Feedstock Monitoring. Another example application is in the protection of pharmaceutical production from contamination of the feedstock. A solvent that is contaminated with another product may significantly affect process yield and efficiency, ultimately costing large sums of money. By monitoring the supply of liquids prior and throughout the process, errant steps may be avoided.

Figure 15 shows the output of a sensor mounted into an IPC flow block and connected to an ethanol supply line. Contamination of the ethanol with water at regular intervals causes the clear steps in sensor output to jump. The large changes shown here make it clear that very small amounts of water contamination (approximately $0.1 \%$ by volume) may be detected.

\section{Summary}

In this paper we have provided a review of the capabilities of planar waveguide Bragg sensors. Starting with the background physical principles we have shown how different types of refractive index sensor may be considered to operate on common principles and then indicated the advantages 


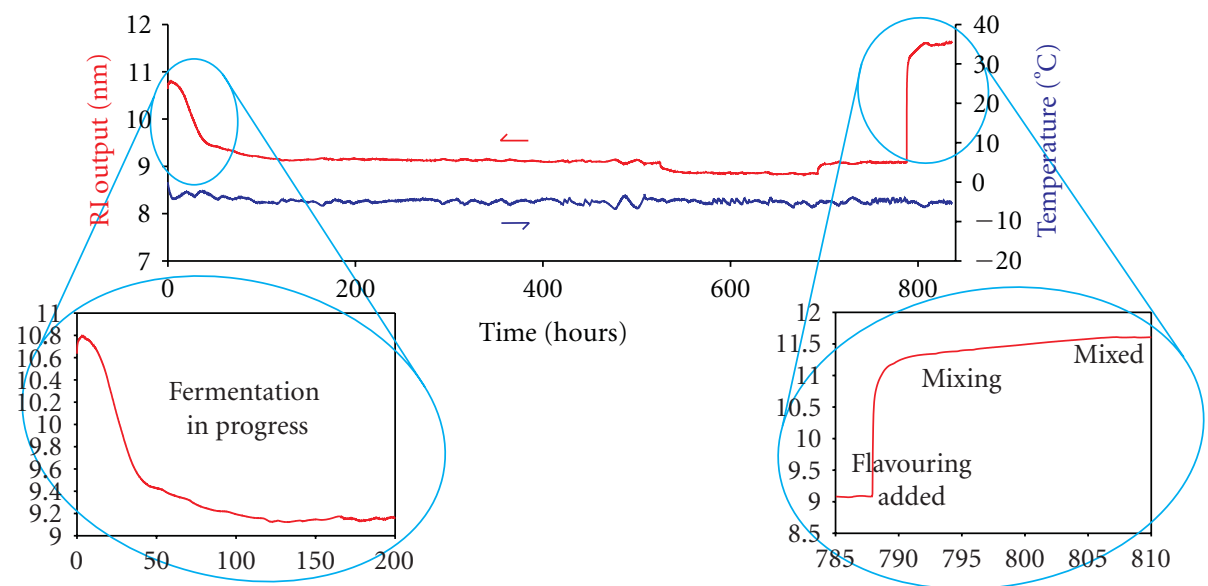

FIGURE 14: Sensor measurement of refractive index and temperature over several weeks during fermentation process. Close-up sections show the initial consumption of sugars and the addition of chemical flavouring.

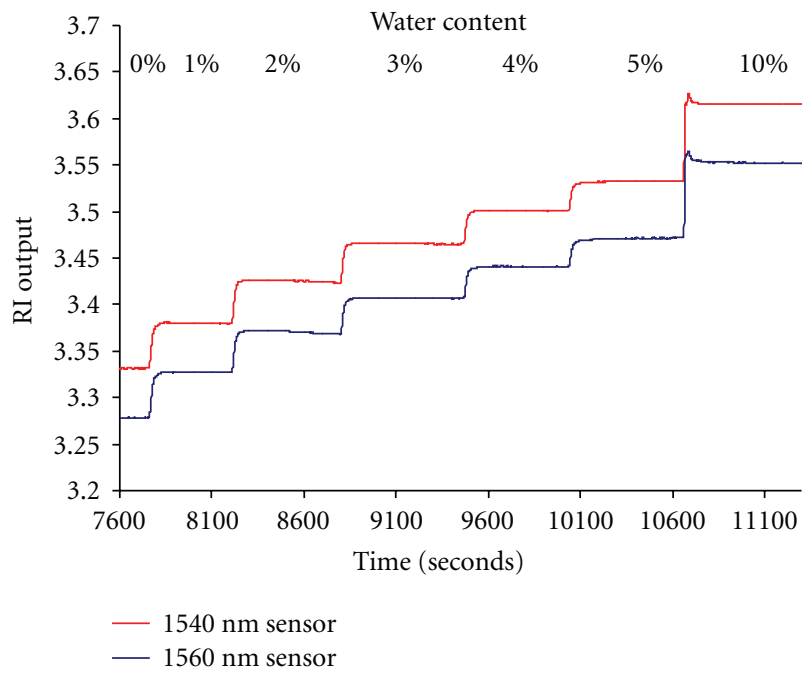

FIGURE 15: Sensor output as ethanol with small variable water content flows over sensing region.

of using a Bragg grating-based device using a modal light confinement approach. We have reviewed in detail the fabrication and packaging routes for such devices and given examples of their behaviour in a range of industrial applications. Data presented shows refractive index sensitivity of order $1.9 \times 10^{-6}$, and corresponding temperature resolution of $0.03^{\circ} \mathrm{C}$. The devices offer the advantages of temperature self-referencing and a microfluidic based silica-on-silicon platform.

\section{References}

[1] X. Fan, I. M. White, S. I. Shopova, H. Zhu, J. D. Suter, and Y. Sun, "Sensitive optical biosensors for unlabeled targets: a review," Analytica Chimica Acta, vol. 620, no. 1-2, pp. 8-26, 2008.

[2] P. Dumais, C. L. Callender, J. P. Noad, and C. J. Ledderhof, "Microchannel-based refractive index sensors monolithically integrated with silica waveguides: structures and sensitivities," IEEE Sensors Journal, vol. 8, no. 5, pp. 457-464, 2008.

[3] J. Ctyroky, F. Abdelmalek, W. Ecke, and K. Usbeck, "Modelling of the surface plasmon resonance waveguide sensor with Bragg grating," Optical and Quantum Electronics, vol. 31, no. 9, pp. 927-941, 1999.

[4] G. Nemova and R. Kashyap, "A compact integrated planarwaveguide refractive-index sensor based on a corrugated metal grating," Journal of Lightwave Technology, vol. 25, no. 8, pp. 2244-2250, 2007.

[5] G. Nemova and R. Kashyap, "Theoretical model of a planar integrated refractive index sensor based on surface plasmonpolariton excitation," Optics Communications, vol. 275, no. 1, pp. 76-82, 2007.

[6] S. A. Maier, Plasmonics: Fundamentals and Applications, Springer, New York, NY, USA, 2007.

[7] B. Culshaw and A. Kersey, "Fiber-optic sensing: a historical perspective," Journal of Lightwave Technology, vol. 26, no. 9, pp. 1064-1078, 2008.

[8] R. G. Heideman, G. J. Veldhuis, E. W. H. Jager, and P. V. Lambeck, "Fabrication and packaging of integrated chemooptical sensors," Sensors and Actuators B, vol. 35, no. 1-3, pp. 234-240, 1996.

[9] K. Tiefenthaler and W. Lukosz, "Integrated optical switches and gas sensors," Optics Letters, vol. 9, no. 4, pp. 137-139, 1984.

[10] K. Schroeder, W. Ecke, R. Mueller, R. Willsch, and A. Andreev, "A fibre Bragg grating refractometer," Measurement Science and Technology, vol. 12, no. 7, pp. 757-764, 2001.

[11] F. Ouerghi, W. Belhadj, F. Abdelmalek, M. Mejatty, and H. Bouchriha, "Polymer thin films and Bragg grating structures based temperature and pressure integrated effects," Thin Solid Films, vol. 485, no. 1-2, pp. 176-181, 2005.

[12] C. Wochnowski, M. Abu-El-Qomsan, W. Pieper, et al., "UVlaser assisted fabrication of Bragg sensor components in a planar polymer chip," Sensors and Actuators A, vol. 120, no. 1, pp. 44-52, 2005.

[13] C. Wochnowski, M. T. Kouamo, W. Pieper, et al., "Fabrication of a planar polymeric deformation Bragg sensor component by excimer laser radiation," IEEE Sensors Journal, vol. 6, no. 2, pp. 331-338, 2006. 
[14] K. J. Kim and M. C. Oh, "Flexible Bragg reflection waveguide devices fabricated by post-lift-off process," IEEE Photonics Technology Letters, vol. 20, no. 1-4, pp. 288-290, 2008.

[15] T. Pustelny, I. Zielonka, C. Tyszkiewicz, P. Karasinski, and B. Pustelny, "Impressing technology of optical Bragg's gratings on planar optical sol-gel waveguides," Opto-Electronics Review, vol. 14, no. 2, pp. 161-166, 2006.

[16] V. M. N. Passaro, R. Loiacono, et al., "Design of Bragg grating sensors based on submicrometer optical rib waveguides in SOI," IEEE Sensors Journal, vol. 8, no. 9-10, pp. 1603-1611, 2008.

[17] S.-L. Tsao and C.-P. Peng, "An SOI Michelson interferometer sensor with waveguide Bragg reflective gratings for temperature monitoring," Microwave and Optical Technology Letters, vol. 30, no. 5, pp. 321-322, 2001.

[18] D. Runde, S. Brunken, C. E. Ruter, and D. Kip, "Integrated optical electric field sensor based on a Bragg grating in lithium niobate," Applied Physics B, vol. 86, no. 1, pp. 91-95, 2007.

[19] I. J. G. Sparrow, G. D. Emmerson, C. B. E. Gawith, and P. G. R. Smith, "Planar waveguide hygrometer and state sensor demonstrating supercooled water recognition," Sensors and Actuators B, vol. 107, no. 2, pp. 856-860, 2005.

[20] I. J. G. Sparrow, G. D. Emmerson, C. B. E. Gawith, P. G. R. Smith, M. Kaczmarek, and A. Dyadyusha, "First order phase change detection using planar waveguide Bragg grating refractometer," Applied Physics B, vol. 81, no. 1, pp. 1-4, 2005.

[21] X. Dai, S. J. Mihailov, C. L. Callender, C. Blanchetiere, and R. B. Walker, "Ridge-waveguide-based polarization insensitive Bragg grating refractometer," Measurement Science \& Technology, vol. 17, no. 7, pp. 1752-1756, 2006.

[22] S.-M. Lee, K.-C. Ahn, and J. S. Sirkis, "Planar optical waveguide temperature sensor based on etched Bragg gratings considering nonlinear thermo-optic effect," KSME International Journal, vol. 15, no. 3, pp. 309-319, 2001.

[23] A. V. Dotsenko, A. L. Diikov, and T. A. Vartanyan, "Labelfree biosensor using an optical waveguide with induced Bragg grating of variable strength," Sensors and Actuators B, vol. 94, no. 1, pp. 116-121, 2003.

[24] H. Nishiyama, Y. Hirata, I. Miyamoto, and J. Nishii, "Formation of periodic structures by the space-selective precipitation of Ge nanoparticles in channel waveguides," Applied Surface Science, vol. 253, no. 15, pp. 6550-6554, 2007.

[25] M. Svalgaard, K. Faerch, and L.-U. Andersen, "Variable optical attenuator fabricated by direct UV writing," Journal of Lightwave Technology, vol. 21, no. 9, pp. 2097-2103, 2003.

[26] P. Cheben, E. Post, S. Janz, et al., “Tilted fiber Bragg grating sensor interrogation system using a high-resolution siliconon-insulator arrayed waveguide grating," Optics Letters, vol. 33, no. 22, pp. 2647-2649, 2008.

[27] A. J. Willshire, P. Niewczas, and J. R. Mcdonald, "A cyclic arrayed-waveguide-grating-based fiber-optic sensor interrogation system," IEEE Photonics Technology Letters, vol. 18, no. 18, pp. 1904-1906, 2006.

[28] G. Z. Xiao, P. Zhao, F. G. Sun, Z. G. Lu, Z. Zhang, and C. P. Grover, "Interrogating fiber Bragg grating sensors by thermally scanning a demultiplexer based on arrayed waveguide gratings," Optics Letters, vol. 29, no. 19, pp. 2222-2224, 2004.

[29] G. D. Emmerson, S. P. Watts, C. B. E. Gawith, et al., "Fabrication of directly UV-written channel waveguides with simultaneously defined integral Bragg gratings," Electronics Letters, vol. 38, no. 24, pp. 1531-1532, 2002.
[30] C. B. E. Gawith, G. D. Emmerson, S. G. McMeekin, et al., "Small-spot interference pattern for single-step 2D integration and wide wavelength detuning of planar Bragg gratings," Electronics Letters, vol. 39, no. 14, pp. 1050-1051, 2003.

[31] R. Feced, M. N. Zervas, and M. A. Muriel, "An efficient inverse scattering algorithm for the design of nonuniform fiber Bragg gratings," IEEE Journal of Quantum Electronics, vol. 35, no. 8, pp. 1105-1115, 1999.

[32] G. R. Quigley, R. D. Harris, and J. S. Wilkinson, "Sensitivity enhancement of integrated optical sensors by use of thin highindex films," Applied Optics, vol. 38, no. 28, pp. 6036-6039, 1999. 

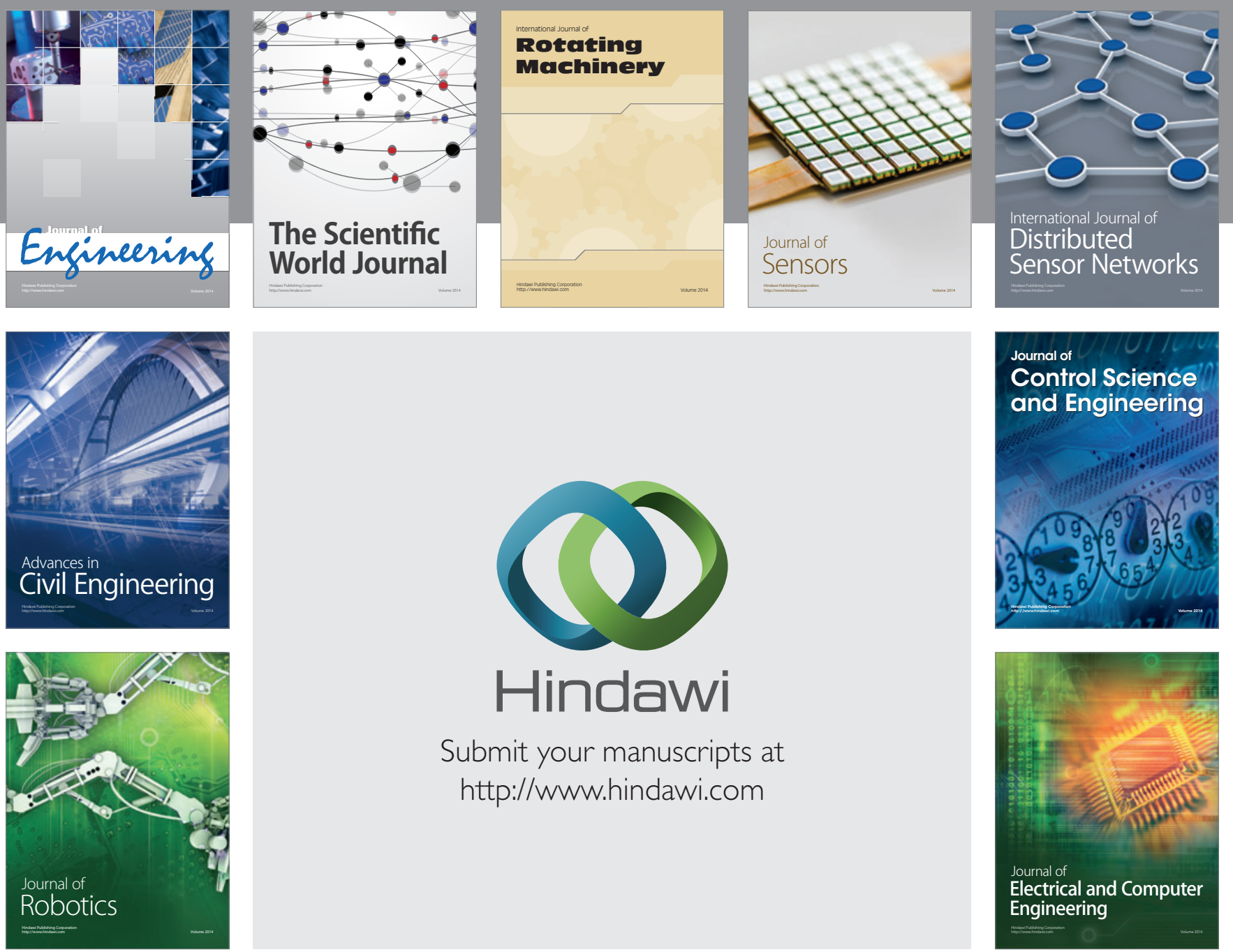

Submit your manuscripts at

http://www.hindawi.com
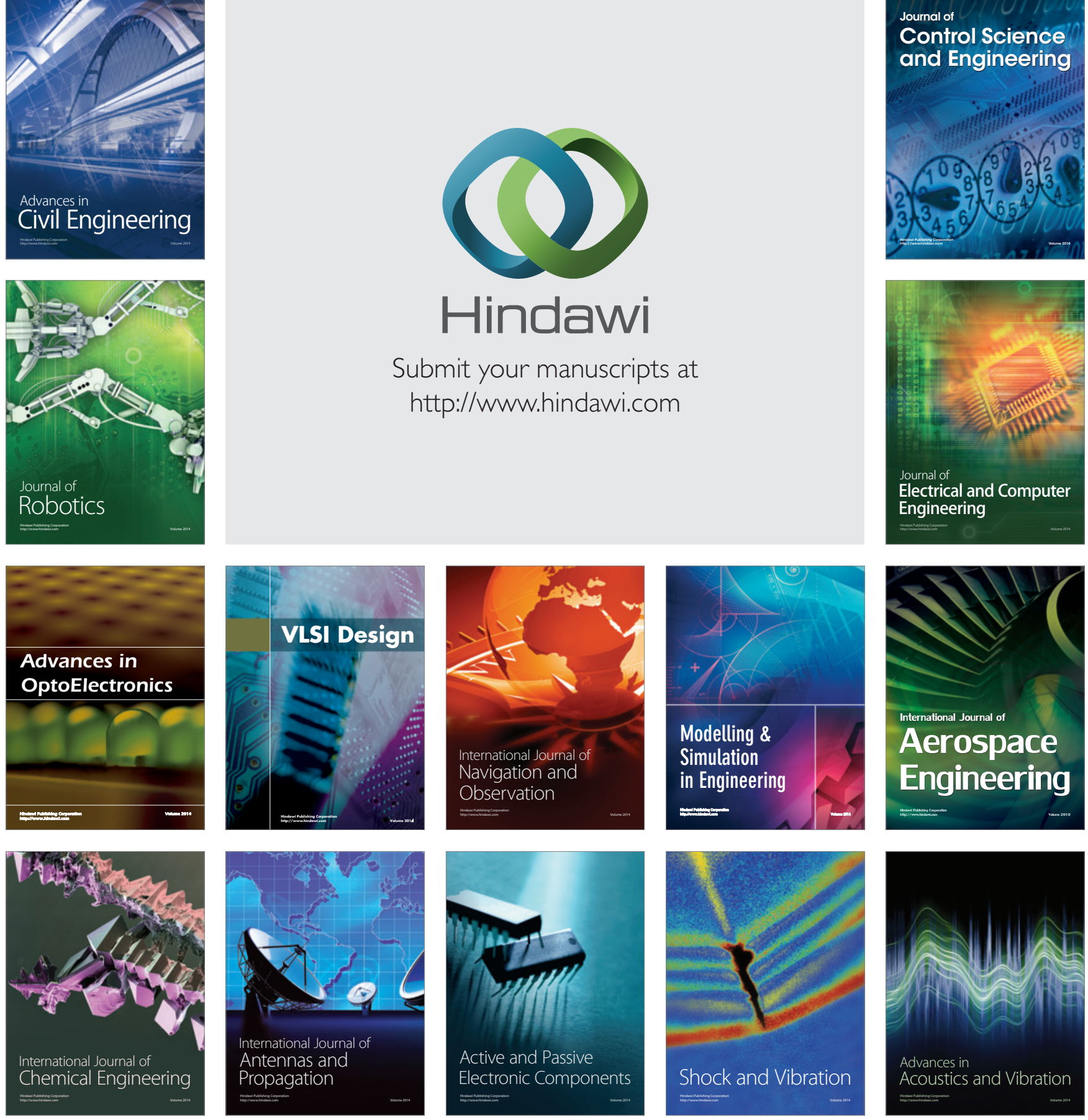